\title{
A Two-Step Adhesion Cascade for T Cell/Endothelial Cell Interactions under Flow Conditions
}

\author{
David A. Jones, * Larry V. McIntire, * C. Wayne Smith, ${ }^{\star}$ and Louis J. Picker $\$$ \\ *Cox Laboratory for Biomedical Engineering, Institute of Biosciences and Bioengineering, Department of Chemical Engineering, Rice \\ University, Houston, Texas 77251-1892; ${ }^{\ddagger}$ Speros P. Martel Laboratory of Leukocyte Biology, Department of Pediatrics, Department of \\ Microbiology and Immunology, Baylor College of Medicine, Houston, Texas 77030; and ${ }^{\S}$ Laboratory of Molecular Pathology, \\ Department of Pathology, University of Texas Southwestern Medical Center, Dallas, Texas 75235-9072
}

\begin{abstract}
Neutrophil adherence to endothelial cells (ECs) under conditions of flow occurs in successive steps, including selectindependent primary adhesion and CD18-dependent secondary adhesion. We used a parallel-plate flow chamber to assess the steps in $T$ cell adherence in vitro. On monolayers of $L$ cells transfected with the $\mathrm{EC}$ adhesion molecules $\mathrm{E}$ selectin, vascular cell adhesion molecule-1 (VCAM-1), or intercellular adhesion molecule-1 (ICAM-1), E-selectin was capable of mediating only primary adhesion, ICAM-1 was capable of mediating only secondary adhesion, and VCAM1 was capable of mediating both primary and secondary adhesion. Studies using human umbilical vein EC monolayers stimulated for $24 \mathrm{~h}$ with IL-1 also revealed distinct primary and secondary steps in $\mathbf{T}$ cell adhesion under flow, and the secondary adhesion was inhibited $>90 \%$ by blocking both VCAM-1/ $\alpha_{4} \beta_{1}$ integrin and ICAM-1/CD18 integrin pathways. However, the primary adhesion under conditions of flow could not be attributed to any of the mechanisms known to support adhesion of leukocytes to ECs. Alone, this pathway was shown to mediate $T$ cell rolling and was a necessary prerequisite for engagement of the two integrin pathways in this system. Thus, $T$ cell adherence to 24-h IL-1-stimulated human umbilical vein ECs at venular wall shear stresses involves at least two successive steps, with clear molecular distinctions from the mechanisms accounting for neutrophil/EC adhesion. (J. Clin. Invest. 1994. 94:2443-2450.) Key words: cell adhesion molecules • vascular endothelium • lymphocytes • interleukin-1 • monoclonal antibodies
\end{abstract}

Address correspondence to David A. Jones, Cox Laboratory for Biomedical Engineering, Institute of Biosciences and Bioengineering, Rice University, Houston, TX 77251-1892.

Received for publication 29 March 1994 and in revised form 5 August 1994.

1. Abbreviations used in this paper: CLA, cutaneous lymphocyte antigen; D-PBS, Dulbecco's phosphate-buffered saline; EC, endothelial cell; HUVEC, human umbilical vein EC; ICAM-1, intercellular adhesion molecule-1; L-ELAM, murine L cells expressing human E-selectin; LICAM-1; ICAM-1-transfected L cells; L-VCAM-1, L cells transfected with the seven-domain form of VCAM-1; MAdCAM-1, mucosal addressin cell adhesion molecule-1; VCAM-1, vascular cell adhesion molecule-1.

J. Clin. Invest.

(C) The American Society for Clinical Investigation, Inc. $0021-9738 / 94 / 12 / 2443 / 08 \quad \$ 2.00$

Volume 94, December 1994, 2443-2450

\section{Introduction}

Detailed studies of acute inflammatory processes suggest that neutrophil/endothelial cell (EC) ${ }^{1}$ interaction can be divided into several successive steps. These steps include primary adhesion, in which free-flowing neutrophils bind and roll slowly along the surface of activated endothelium, secondary firm adhesion of the rolling cells, and subsequent transmigration. Distinct families of adhesion molecules have been shown to participate in the primary and secondary adhesive events for neutrophils. Primary adhesion involves all three members of the selectin family (1-5) and their oligosaccharide ligands, while secondary adhesion involves $\beta_{2}(\mathrm{CD} 18)$ integrins on neutrophils (1) and immunoglobulin gene superfamily counterreceptors on EC (primarily intercellular adhesion molecule-1 [ICAM-1]) (2). Selectin-ligand interactions are activation independent, are rapid enough to bind free-flowing neutrophils, and appear to break in such a way as to allow adherent leukocytes to roll slowly along the endothelium $(6,7)$. In contrast, CD18 integrin-ligand interactions are activation dependent (i.e., high avidity can be induced through a conformation change), are not able to initiate adhesion under flow conditions, but can mediate highly stable adhesion under static conditions or once binding is initiated by selectins $(1,4,8,9)$.

Fewer details are known about the interactions between lymphocytes and EC during extravasation at sites of inflammation. The selectin and CD18 integrin adhesion pathways, as well as other pathways not used by neutrophils, including the $\alpha_{4} \beta_{1}$ integrin (VLA-4) and $\alpha_{4} \beta_{7}$ integrin pathways (which preferentially bind to the EC ligands vascular cell adhesion molecule1 [VCAM-1] and mucosal addressin cell adhesion molecule-1 [MAdCAM-1], respectively), have been implicated in lymphocyte-EC recognition $(10,11)$. However, most studies of the adhesive functions of these pathways have used assays which do not discriminate the relative participation of these pathways in primary and secondary adhesion. It is therefore still not clear if lymphocyte-EC adhesive interactions under flow are analogous to the multistep process described for neutrophils, and if so, which receptors contribute at each stage.

This study addresses these issues by examining the behavior of purified peripheral blood $\mathrm{T}$ cells in static and flow adhesion assays using two model systems: $(a)$ murine L cell monolayers transfected with a single human receptor (E-selectin, ICAM-1, or VCAM-1) to examine adhesion mediated by single adhesion pathways; and $(b)$ cultured human umbilical vein EC (HUVEC) monolayers stimulated with cytokines to explore possible cooperative interactions between the various adhesion molecule pairs in a physiologically relevant cell type. Monoclonal antibody $(\mathrm{mAb})$ blocking was used to confirm the specificity of interaction using the transfected cells and to analyze the contri- 
butions of particular adhesion pathways with the HUVECs. Our results confirm that $T$ cell interactions with 24-h IL-1-stimulated HUVEC monolayers involve distinct primary and secondary adhesion steps and use both ICAM-1/CD18 integrin and VCAM-1/ $\alpha_{4} \beta_{1}$ integrin interactions. However, we were unable to define the adhesion molecules that account for primary adhesion of $\mathrm{T}$ cells to activated $\mathrm{EC}$ monolayers and suggest that this event may be independent of the three known members of the selectin family.

\section{Methods}

Isolation of $T$ lymphocytes. Human peripheral blood $\mathrm{T}$ cells were obtained by centrifugation of whole blood through Ficoll-Hypaque (Histopaque 1077; Sigma Chemical Co., St. Louis, MO) and negative selection using a T cell enrichment column (R\&D Systems, Inc., Minneapolis, $\mathrm{MN})$. Preparations were $>95 \% \mathrm{CD}^{+}$and $>99 \%$ viable and were labeled when necessary using the fluorescent DNA-binding dye Hoescht 33342 (bis-benzamide; Sigma Chemical Co.; reference 12). Chymotrypsin treatment to remove surface L-selectin was performed as described elsewhere (13). Cutaneous lymphocyte antigen (CLA) ${ }^{+}$ $\mathrm{CD} 4 \mathrm{RA}^{-}$and $\mathrm{CLA}^{-} \mathrm{CD}^{-}$45RA ${ }^{-} \mathrm{T}$ cell populations were prepared using a FACStar ${ }^{\text {Plus }}$ flow cytometer (Becton Dickinson, Mountain View, CA), as described previously (14).

Tissue culture. HUVECs were isolated by collagenase treatment according to established techniques, pooled, and plated in fibronectincoated T75 tissue culture flasks (2). Monolayers were cultured in M199 (Gibco Laboratories, Grand Island, NY) supplemented with 10\% FBS, hydrocortisone ( $1 \mu \mathrm{g} / \mathrm{ml}$; Sigma Chemical Co.), low molecular weight heparin ( $1 \mu \mathrm{g} / \mathrm{ml}$; Sigma Chemical Co.), gentamicin ( $25 \mu \mathrm{g} / \mathrm{ml}$; Sigma Chemical Co.), and amphotericin B ( $1.25 \mu \mathrm{g} / \mathrm{ml}$ as Fungizone; Gibco Laboratories). No growth factors were used. Cells to be used in adhesion assays were passaged 4-7 d after initial seeding using trypsin-EDTA (Sigma Chemical Co.) and plated at confluence on fibronectin-coated 35-mm tissue culture dishes (Corning Glass Works, Corning, NY). Cells for flow cytometry experiments were harvested using EDTA alone. HUVECs were stimulated with recombinant human IL-1 $\beta(10 \mathrm{U} / \mathrm{ml}$; Sigma Chemical Co.), recombinant human IL-4 ( $100 \mathrm{U} / \mathrm{ml}$; R\&D Systems, Inc.), or both before adhesion or flow cytometry studies. ICAM1-transfected L cells (L-ICAM-1) cells were generously provided by Dr. Tim Springer (Center for Blood Research, Boston, MA). Dr. Ted Yednock (Athena Neurosciences, South San Francisco, CA) generously provided $\mathrm{L}$ cells transfected with either the six or seven domain forms of VCAM-1. Except where specifically noted, the seven-domain form (referred to as L-VCAM-1) was used. L-ELAM (L-E-selectin) cells have been described previously (3). Immunofluorescence analysis of receptor expression on HUVECs was performed using standard techniques.

Flow cytometry. For flow cytometry, $\sim 5 \times 10^{4}$ cells per test were incubated with $1 \mu \mathrm{g}$ primary antibody for $30 \mathrm{~min}$, washed, incubated with phycoerythrin-conjugated goat anti-mouse IgG (Sigma Chemical Co.), washed again, and fixed in $1.0 \%$ paraformaldehyde. Data were acquired using a FACScan ${ }^{\circledR}$ flow cytometer and analyzed using PAINT-

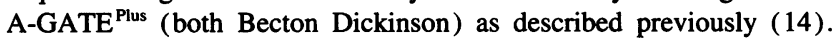
Measurements were made only for single, undisrupted cells by gating according to scatter parameters. DREG56 was used as a nonbinding control mAb in the flow cytometry of HUVECs. Flow cytometry of T cells was performed in the same manner to verify complete removal of L-selectin after chymotrypsin treatment.

Monoclonal antibodies. All monoclonal antibodies were used as purified antibodies. mAbs R6.5 (anti-ICAM-1, CD54) and R15.7 (antiCD18) were provided by Dr. Robert Rothlein (Boehringer Ingelheim Ltd., Ridgefield, CT), and mAb G1 (anti-P-selectin, CD62P) was provided by Dr. Rodger McEver (University of Oklahoma, Oklahoma City, OK). mAb E1/6 (Becton Dickinson) binds VCAM-1 (CD106), mAb HP2/1 (Amac, Inc., Westbrook, ME) binds to the $\alpha_{4}$ chain (CD49d) of $\alpha_{4} \beta_{1}$ integrin (and $\alpha_{4} \beta_{7}$ ), and mAb 5.6E (Amac, Inc.) binds platelet- endothelial cell adhesion molecule-1 (CD31). CL2 (anti-E-selectin, CD62E), DREG56 (anti-L-selectin, CD62L), PJ-18 (binds an irrelevant endothelial cell epitope), Hermes-3 (anti-CD44), MECA-79, and HECA-452 (anti-CLA) have been described previously (14-17).

In static and flow adhesion assays, L cell and HUVEC monolayers were treated with $\mathrm{mAbs}$ for $30 \mathrm{~min}$ at $37^{\circ} \mathrm{C}$ before use. $\mathrm{T}$ cells were treated with $\mathrm{mAbs}$ for $30 \mathrm{~min}$ at room temperature before use. Experiments were also run in which $\mathrm{T}$ cells were treated with mAbs at 37 or $4^{\circ} \mathrm{C}$ instead of at room temperature to check for possible activating or inhibitory side effects of the mAb treatments, and no differences in binding were observed. In most experiments, cells were pretreated with antibodies before use in the flow system, since preliminary experiments showed identical results for pretreatment alone and pretreatment with inclusion of $\mathrm{mAb}$ in the flow buffer.

Adhesion assays. Physiological flow conditions were produced in vitro using a flow chamber with parallel-plate geometry as described previously (2). The chamber produces a well-defined laminar flow over monolayers grown in 35-mm tissue culture dishes and was used for both static and flow adhesion assays.

In the static assays, a suspension of $5 \times 10^{6}$ fluorescently labeled $\mathrm{T}$ cells $/ \mathrm{ml}$ in $37^{\circ} \mathrm{C}$ Dulbecco's phosphate-buffered saline (D-PBS) was infused into the chamber and allowed to settle onto the monolayer for a period of $2 \mathrm{~min}$. All of the T cells settle and contact the monolayer within $1.5 \mathrm{~min}$. Nonadherent cells were removed by flowing D-PBS through the chamber for $15 \mathrm{~s}$ at a wall shear stress of $2.0 \mathrm{dyn} / \mathrm{cm}^{2}$. During all experiments, the flow system was maintained at $37^{\circ} \mathrm{C}$ in a warm air box surrounding the microscope. Images were acquired using videomicroscopy (Diaphot-TMD microscope from Nikon, Inc., Garden City, NY; Hamamatsu C1000 video camera from Hamamatsu Photonics, Bridgewater, NJ) for four fields of view ( $\times 20$ objective; $\sim 0.28 \mathrm{~mm}^{2}$ fields) both before and after rinsing. The average number of cells per field of view after rinsing was divided by that before rinsing to give the percentage of cells adhering for that experiment. Values were then averaged for a minimum of $n=4$ experiments using various $\mathrm{T}$ cell donors and various pools of HUVECs.

In the flow adhesion assay, a suspension of $10^{6} \mathrm{~T}$ cells $/ \mathrm{ml}$ in $\mathrm{D}$ PBS was perfused through the chamber at a wall shear stress of 2.0 $\mathrm{dyn} / \mathrm{cm}^{2}$. A single field of view was monitored during the $10 \mathrm{~min}$ of the experiment, and at the end four fields of view were monitored for $15 \mathrm{~s}$ each. All experiments were videotaped for later analysis (Sony SLV400 VCR; Sony Corp., Park Ridge, NJ). Three quantities were measured in the analysis: the total interacting cells during the entire 10min experiment, the number of stably adherent cells at the end, and the average rolling velocity. The total number of interacting cells is the number of cells which interact with the monolayer (either rolling or immediately arrested) for at least $1 \mathrm{~s}$ and was determined manually by reviewing the videotapes. Stably adherent cells accumulate throughout each experiment and were counted for each of four fields of view at the end. Only cells which remained stationary for at least $5 \mathrm{~s}$ were counted as stably adherent cells. Rolling velocities were measured as described previously (2). Briefly, after $\sim 9 \mathrm{~min}$ of flow we acquired several 4-s "maximization" images (see Fig. 5) using a digital image processing system (Sun Microsystems, Mountain View, CA; Inovision Corp., Durham, NC). The lengths of the blurs divided by the acquisition time (4 s) give the rolling velocity.

In static and flow adhesion assays using $\mathrm{L}$ cell monolayers, $\mathrm{T}$ cells were fluorescently labeled to allow accurate image analysis, while in experiments with HUVEC monolayers $T$ cells were left unlabeled and could be readily distinguished with phase contrast. Control experiments for effects of the labeling were performed using unlabeled $\mathrm{T}$ cells, and no differences were found between labeled and unlabeled $T$ cells.

\section{Results}

Patterns of $T$ cell adhesion to transfectant $L$ cell monolayers. The results of static binding experiments using transfectant cell 


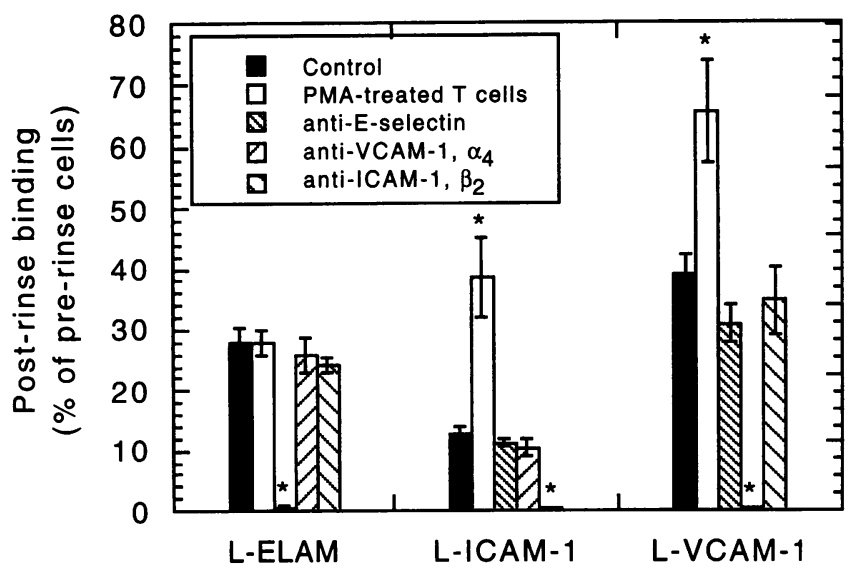

Figure 1. Adhesion of $\mathrm{T}$ cells to transfected $\mathrm{L}$ cells under static conditions. $T$ cells were allowed to settle onto an $L$ cell monolayer for 2 min, then nonadherent cells were rinsed away by flowing D-PBS through the chamber for $15 \mathrm{~s}$ at a wall shear stress of $2.0 \mathrm{dyn} / \mathrm{cm}^{2}$. Adhesion levels are given as post-rinse cells/pre-rinse cells $\times 100 \%$; error bars represent mean \pm SEM for $n=4-6$ experiments. PMA treatment was performed at a concentration of $50 \mathrm{ng} / \mathrm{ml}$ PMA for $30 \mathrm{~min}$ at $37^{\circ} \mathrm{C}$. Treatments with blocking mAbs $(10 \mu \mathrm{g} / \mathrm{ml})$ are described in the text. An asterisk indicates a statistically significant $(P<0.05)$ difference in binding relative to control.

lines are presented in Fig. 1. Anti-E-selectin mAb CL2, antiICAM-1 mAb R6.5, anti- $\beta_{2}$ (anti-CD18) mAb R15.7, antiVCAM-1 mAb E1/6, and anti- $\alpha_{4}$ (anti-CD49d) mAb HP2/1 were found to specifically and completely inhibit $\mathrm{T}$ cell adhesion via the E-selectin, ICAM- $1 / \beta_{2}$ integrin, and VCAM- $1 / \alpha_{4} \beta_{1}$ integrin pathways. In these experiments, integrin pathways were blocked using antibodies to both components of the pathway simultaneously, although adhesion could be completely blocked by any of the antibodies used alone (data not shown). During the 15-s rinse, adherent $\mathrm{T}$ cells rolled along the L-ELAM monolayers, but $\mathrm{T}$ cells remained stationary on the L-ICAM-1 and L-VCAM-1 monolayers, indicating that the static incubation allowed development of secondary adhesion via ICAM-1 and VCAM-1 but not E-selectin.

Fig. 2 shows the results of experiments to determine which of these adhesion pathways support initial attachment under flow conditions (primary adhesion). At $2.0 \mathrm{dyn} / \mathrm{cm}^{2}$ wall shear stress, the L-ELAM and L-VCAM-1 monolayers supported primary adhesion of $T$ cells while the L-ICAM-1 monolayer did not. Here again, the mAbs specifically and completely blocked the appropriate adhesion pathways. Although T cells adhered to both L-ELAM and L-VCAM-1 monolayers under flow, there was an important qualitative difference in this adhesion (Fig. 3 ): the L-ELAM monolayers supported rolling with very little stable adhesion, whereas L-VCAM-1 monolayers supported predominantly an immediate arrest pattern of interaction with $\sim 70 \%$ becoming stably adherent. Some $\mathrm{T}$ cells did roll on the L-VCAM-1 monolayers, but these were fewer than $\sim 10 \%$ of the interacting cells. Adhesion of T cells to L cells expressing the six-domain form of VCAM-1 was essentially identical to results with the seven-domain form (data not shown). We also observed that the $\mathrm{T}$ cells do not appear to be moving unusually slowly when they adhere to the L-VCAM-1 cells, nor do they appear to adhere preferentially to either the leading or trailing edges of these cells, suggesting that topography of the LVCAM-1 monolayers does not influence primary adhesion.

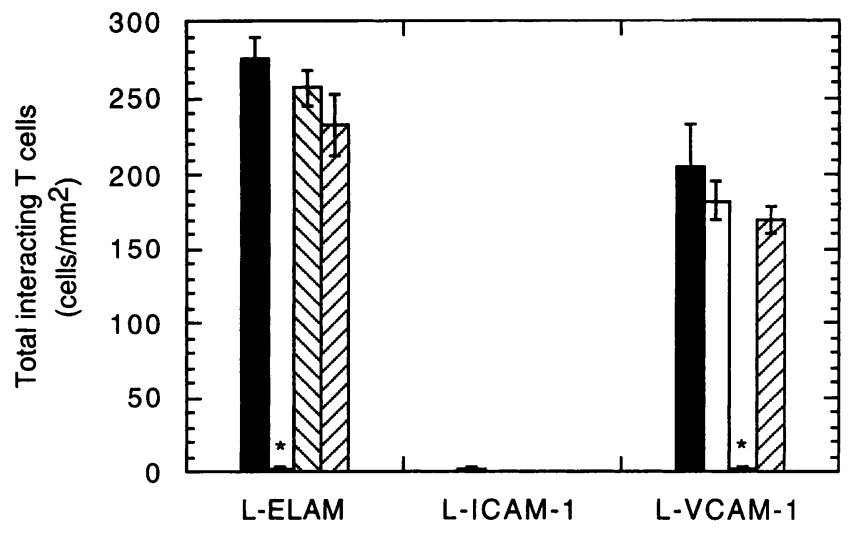

Figure 2. Adhesion of $\mathrm{T}$ cells to transfected $\mathrm{L}$ cells under flow conditions. T cells were perfused at $10^{6}$ cells $/ \mathrm{ml}$ through a parallel-plate flow chamber at a wall shear stress of $2.0 \mathrm{dyn} / \mathrm{cm}^{2}$ for $10 \mathrm{~min}$. T cells which interacted with the monolayers for at least $1 \mathrm{~s}$ were counted throughout the $10 \mathrm{~min}$ of flow and are presented as mean \pm SEM for $n=4-6$ experiments. In the case of adhesion to L-ICAM- 1 cells, there was no adhesion for $n=3$ experiments. Only the appropriate $\mathrm{mAb}$ blocks produced statistically significant differences in binding relative to control $\left({ }^{*}\right)$. . . Control; $\square$, anti-E-selectin; $₫$, anti-VCAM-1, $\alpha_{4} ;$ and $\square$, anti-ICAM- $1, \beta_{2}$.

Under static conditions, PMA significantly increased adhesion mediated by integrin mechanisms, but not by selectin mechanisms (Fig. 1). PMA also increased stable adhesion to L-VCAM-1 monolayers from $\sim 70 \%$ of the interacting cells to $\sim 90 \%$, but did not increase primary adhesion of the stimulated T cells to L-ICAM-1 or L-VCAM-1 monolayers under flow (Fig. 3).

We also performed experiments using L-ELAM monolayers and memory/effector $T$ cells sorted according to expression of the major T cell E-selectin ligand CLA $(14,18,19)$ (characterized by mAb HECA-452) and CD45RA. The CLA ${ }^{-} /$CD $^{4} 5 \mathrm{RA}^{-}$

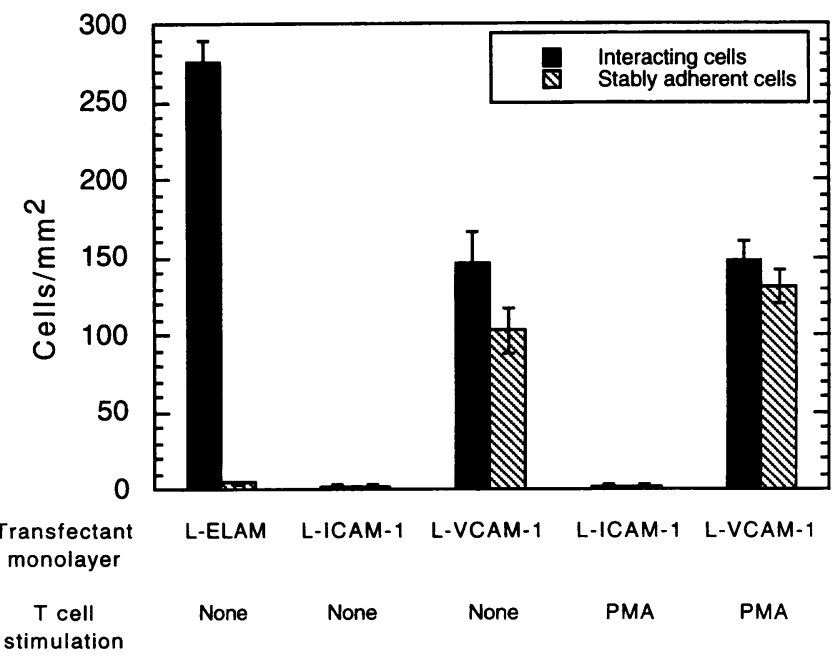

Figure 3. Adhesion of $\mathrm{T}$ cells to transfected $\mathrm{L}$ cells under flow conditions. Experiments were performed as in Fig. 2. Shaded bars represent the total number of cells which interacted with the monolayer for at least $1 \mathrm{~s}$ during a 10-min experiment. Hatched bars show the number of cells which were stationary for at least $5 \mathrm{~s}$ at the end of the 10-min experiment. 
Table I. Flow Cytometric Analysis of Adhesion Receptor Expression

\begin{tabular}{|c|c|c|c|c|c|c|c|}
\hline Cells & Control mAb & Anti-E-selectin & Anti-ICAM-1 & Anti-VCAM-1 & Anti-P-selectin & Anti-CD31 & MECA-79 \\
\hline Unstimulated HUVECs & 29.2 & 14.0 & 121.0 & 88.3 & 28.9 & 191 & 25.2 \\
\hline 4-h IL-1 HUVECs & 21.5 & 222.0 & 196.0 & 394.0 & - & - & 16.2 \\
\hline 24-h IL-1 HUVECs & 16.5 & 65.6 & 469.0 & 521.0 & 21.0 & 243 & 21.3 \\
\hline 24-h IL-1 + IL-4 HUVECs & 9.0 & 33.1 & 359.0 & 959.0 & 36.3 & 188 & 16.0 \\
\hline L-ELAM & 12.0 & 138.0 & 6.8 & 6.6 & - & - & - \\
\hline L-ICAM-1 & 9.7 & 9.9 & 110.0 & 9.5 & - & - & - \\
\hline L-VCAM-1 & 6.2 & 6.5 & 6.3 & 173.0 & - & - & - \\
\hline
\end{tabular}

Values shown are mean fluorescence intensities for which all instrument settings were kept constant. Relative magnitudes are representative of a total of $n=5$ experiments using different batches of cells each time. Background fluorescence was $\sim 6$ for L cells and 9 for HUVECs.

subpopulation bound at very low levels $(15.0 \pm 1.7$ interacting $\mathrm{T}$ cells $\left./ \mathrm{mm}^{2}, n=3\right)$, and the $\mathrm{CLA}^{+} / \mathrm{CD} 45 \mathrm{RA}^{-}$subpopulation adhered (rolling interaction) extensively (501.1 \pm 16.1 interacting $\mathrm{T}$ cells $/ \mathrm{mm}^{2}, n=3$ ), in keeping with previous studies using nonflow assays (19).

$T$ cell adhesion to HUVEC monolayers. The results of flow cytometric analysis of receptor expression on HUVECs and transfectant L cells are given in Table I. The E-selectin, ICAM1 , and VCAM-1 data are in good agreement with previously published results (20-23). HUVECs did not stain with antiP-selectin mAb G1 after IL-1 or IL-1 + IL-4 stimulation for $24 \mathrm{~h}$, although we have previously used this mAb to demonstrate P-selectin expression soon after histamine stimulation (2).

In adhesion experiments under static conditions (Fig. 4), there was no apparent contribution of E-selectin, consistent with its low level of expression on 24-h stimulated HUVECs (Table I). On 24-h IL-1 -stimulated HUVECs, there were comparable contributions of the VCAM-1 and ICAM-1 pathways, but on IL-

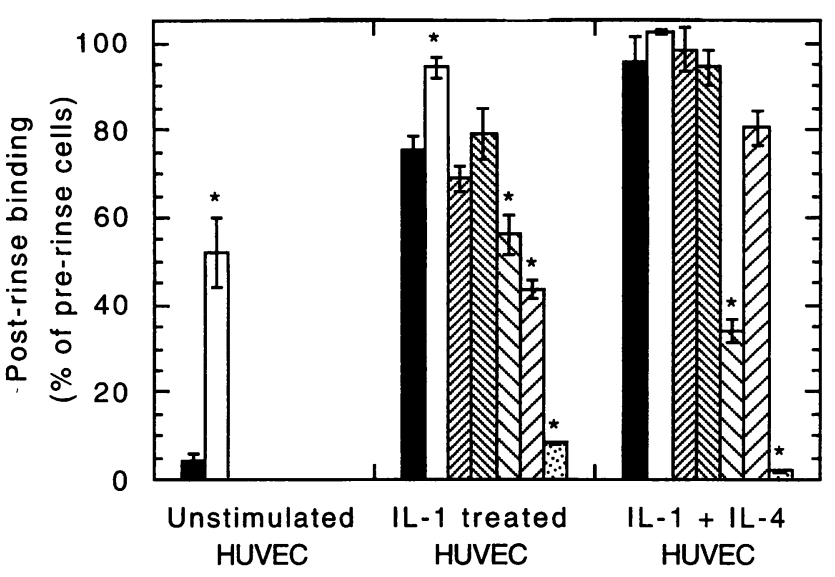

Figure 4. Adhesion of T cells to HUVECs under static conditions. Experiments were performed as in Fig. 1. HUVECs were left untreated or treated for $24 \mathrm{~h}$ with $10 \mathrm{U} / \mathrm{ml}$ IL-1 or IL-1 plus $100 \mathrm{U} / \mathrm{ml} \mathrm{IL-4.}$ Control binding was measured with no mAb treatments but we also performed experiments using mAb PJ-18, which binds an irrelevant HUVEC epitope, and observed similar binding to experiments without mAbs (data not shown). An asterisk indicates a statistically significant $(P<0.05)$ change in binding level relative to control. 1 , Control; $\square$, PMA-treated T cells; $\square$, anti-E-selectin; $₫$, anti-L-selectin; $\varangle$, anti-VCAM- $1, \alpha_{4} ; \nabla$, anti-ICAM- $1, \beta_{2}$; and $\circledast$, anti-VCAM- $1, \alpha_{4}$, ICAM-1, $\beta_{2}$.
$1+$ IL-4-stimulated HUVECs, the VCAM-1 pathway appeared predominant, a finding consistent with enhanced expression of VCAM-1 on these monolayers (Table I).

Under flow conditions, we observed substantial heterogeneity in the qualitative binding patterns of T cells to HUVEC monolayers. Some $\mathrm{T}$ cells contacted the monolayer and rolled for some distance, which was variable but sometimes quite extended (at least the 500- $\mu \mathrm{m}$ length of a field of view). Rolling was followed by release into the free stream or arrest. Arrest was often brief and followed by more rolling, but also resulted in long-term stable adhesion. Additionally, we observed an "immediate arrest" pattern of adhesion in which some $\mathrm{T}$ cells contacted the monolayer and were arrested with no obvious period of rolling. Immediate arrest, like rolling, was transient or stable. These observations are in agreement with reported binding interactions in vivo $(24,25)$. Since immediate arrest is often transient, the observed binding patterns can be interpreted in terms of a multistep model if the definition of primary adhesion is not limited to a rolling interaction but is more broadly defined as an interaction which is able to initiate adhesion of leukocytes moving with the bloodstream. Similarly, secondary adhesion is not defined in terms of cellular arrest only, but rather as adhesion which mediates a relatively long-term, stable arrest. Primary adhesion is quantified as the total interacting cells during a 10-min experiment, and secondary adhesion is quantified as the number of stably adherent cells which have accumulated at the end. The amount of secondary adhesion measured in the flow assay depends on the amount of prerequisite primary adhesion, and secondary adhesion in the absence of primary adhesion mechanisms can only be measured in the static assay. Fig. 5 presents some example images to illustrate what is seen by videomicroscopy in the static and flow adhesion assays. Adhesion of T cells to HUVECs stimulated for $24 \mathrm{~h}$ with IL-1 was substantially reduced at $4.0 \mathrm{dyn} / \mathrm{cm}^{2}$ wall shear stress relative to $2.0 \mathrm{dyn} / \mathrm{cm}^{2}$ ( $\sim 50 \%$ less ). Adhesion behavior was similar in both cases: many cells rolled for some distance before arrest, others rolled without stopping, and others were arrested immediately upon contact. A similar rapid decrease in adhesion with increasing shear stress has been reported for neutrophils $(2,4,26)$.

Quantitation of adhesion under flow revealed that $\sim 50 \%$ of the T cells interacting with 24-h IL-1-stimulated HUVECs became stably adherent (Fig. 6). PMA stimulation of the T cells reduced primary adhesion somewhat and markedly altered the nature of the interactions, promoting immediate arrest that remained stable. Primary adhesion was not significantly reduced 

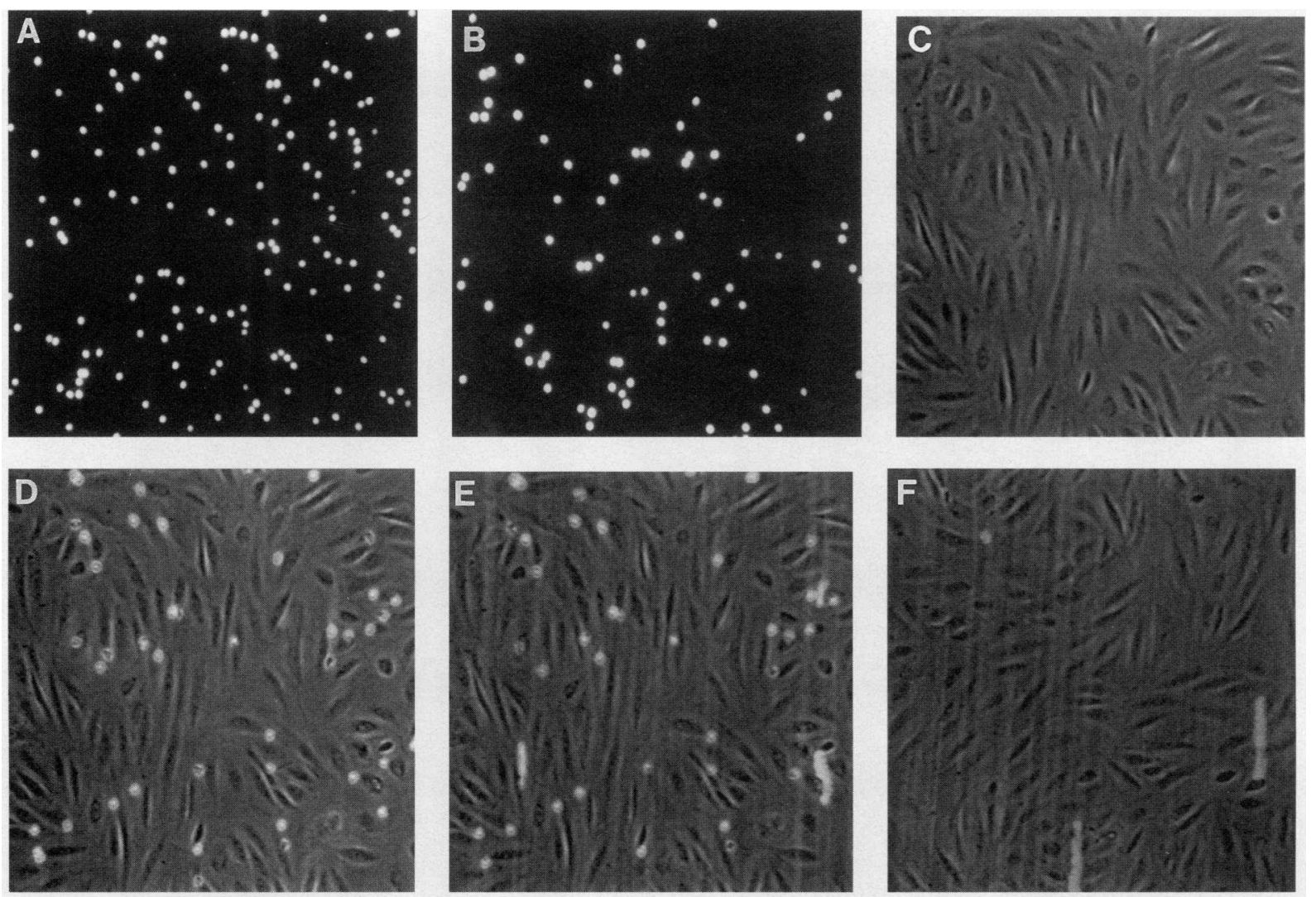

Figure 5. Static and flow adhesion assays. $A$ and $B$ are example images of the static assay before and after rinsing nonadherent cells. $C$ and $D$ are example images of the flow adhesion assay. $C$ shows a monolayer of 24-h IL-1-stimulated HUVECs before flow, and $D$ shows the same monolayer after $10 \mathrm{~min}$ of continuous flow of T cells $\left(10^{6} \mathrm{cells} / \mathrm{ml}\right)$ through the chamber at $2.0 \mathrm{dyn} / \mathrm{cm}^{2} . E$ is a maximization image of $\mathrm{T}$ cells adhering to 24-h IL-1-stimulated HUVECs which is analogous to a 4-s photographic exposure. The blurs are the paths of two $\mathrm{T}$ cells which rolled along the monolayer during the $4 \mathrm{~s}$ of image acquisition. Images such as these were used to calculate rolling velocities. $F$ is similar to $E$, except that in this case mAbs which block the VCAM- $1 / \alpha_{4} \beta_{1}$ integrin and ICAM- $1 / \alpha_{\mathrm{L}} \beta_{2}$ integrin adhesion pathways were used. T cells rolled along the ECs under these conditions, but even after $10 \mathrm{~min}$ of flow very few established firm adhesion. Rolling velocity increased with blockade of the integrin pathways as well.

by any of the blocking mAbs (Fig. 7), and using anti-L-selectin and anti-E-selectin mAbs together also had no significant effect (data not shown). Additional studies were carried out to assess a possible role for $\mathrm{L}$-selectin. Treatment of the $\mathrm{T}$ cells with chymotrypsin to remove surface L-selectin failed to reduce the primary adhesion, and treatment of 24-h IL-1-stimulated HUVECs with L-selectin-Ig chimera (generously supplied by Dr. S. Watson, Genentech Inc., South San Francisco, CA) at concentrations of 10 and $100 \mu \mathrm{g} / \mathrm{ml}$ (27) also failed to reduce primary adhesion. Stimulated HUVECs did not stain with mAb MECA-79 (Table I), which detects the high affinity L-selectin ligands expressed by high endothelial venules in peripheral lymph node and other sites (17). L-selectin lymphoid cell transfectants also failed to adhere to the stimulated HUVECs in the flow assay (data not shown).

Secondary adhesion to 24-h IL-1-stimulated HUVECs was inhibited by $\sim 90 \%$ using mAbs which block the $\alpha_{4} \beta_{1}$ integrin/ VCAM-1 and $\beta_{2}$ integrin/ICAM-1 pathways (Fig. 7). Most interacting cells in these experiments rolled along the HUVEC monolayers. Stimulation with both IL-1 and IL-4 completely prevented primary adhesion of $\mathrm{T}$ cells (Fig. 6), although the secondary adhesion mechanisms seemed to be intact since these monolayers supported high levels of both VCAM-1 - and ICAM-1-dependent adhesion of $\mathrm{T}$ cells under static conditions
(Fig. 4). VCAM-1 is highly expressed on these cells (Table I), so the lack of primary adhesion contrasts with the primary adhesion seen with the L-VCAM-1 monolayers. Surface distribution does not appear to explain this difference since immunofluorescence studies of HUVECs and L cells showed that VCAM-1 has a relatively uniform surface distribution on both L cells and stimulated HUVECs.

$T$ cell rolling velocities. Fig. 8 presents $\mathrm{T}$ cell rolling velocities under various conditions. These $T$ cell rolling velocities agree well with previously reported lymphocyte rolling velocities in vivo (28) as well as previously reported neutrophil rolling velocities both in vitro $(2,3,5)$ and in vivo $(29,30)$. Blockade of each integrin pathway increased rolling velocity, similar to the effect of CD18 integrin/ICAM-1 interactions on rolling velocity which has been reported for neutrophils $(2,8$, 9, 31).

\section{Discussion}

A cornerstone of the multistep model of neutrophil extravasation is the observation that primary and secondary adhesion under conditions of flow are mediated by distinct classes of adhesion molecules: the selectins and their carbohydrate-containing ligands for primary adhesion, and the $\beta_{2}$ integrins and 


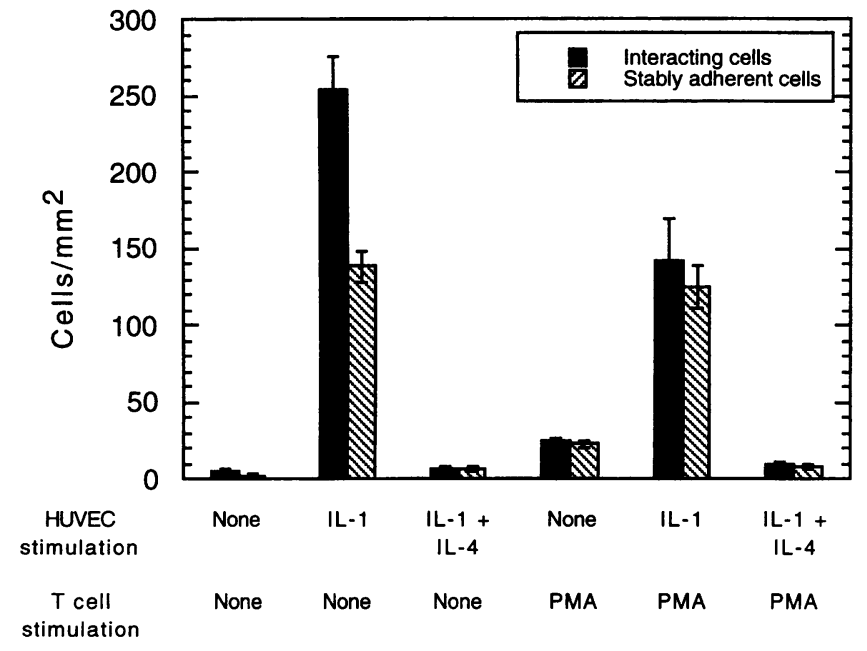

Figure 6. Adhesion of T cells to HUVECs under flow conditions: effects of various cell stimuli. Note that the lack of adhesion to IL-1 + IL-4treated HUVECs under flow is in marked contrast to the very high adhesion observed under static conditions.

their immunoglobulin superfamily ligands (primarily ICAM-1) for secondary adhesion (1-3). To begin investigating this issue with lymphocytes, we examined the binding of isolated $\mathrm{T}$ cells to transfected $\mathrm{L}$ cell monolayers (E-selectin, ICAM-1, or VCAM-1 transfectants) under both static and flow conditions. Under static conditions, $\mathbf{T}$ cells developed adhesion to all three transfectant monolayers, and this binding was completely inhibited by appropriate blocking mAbs.

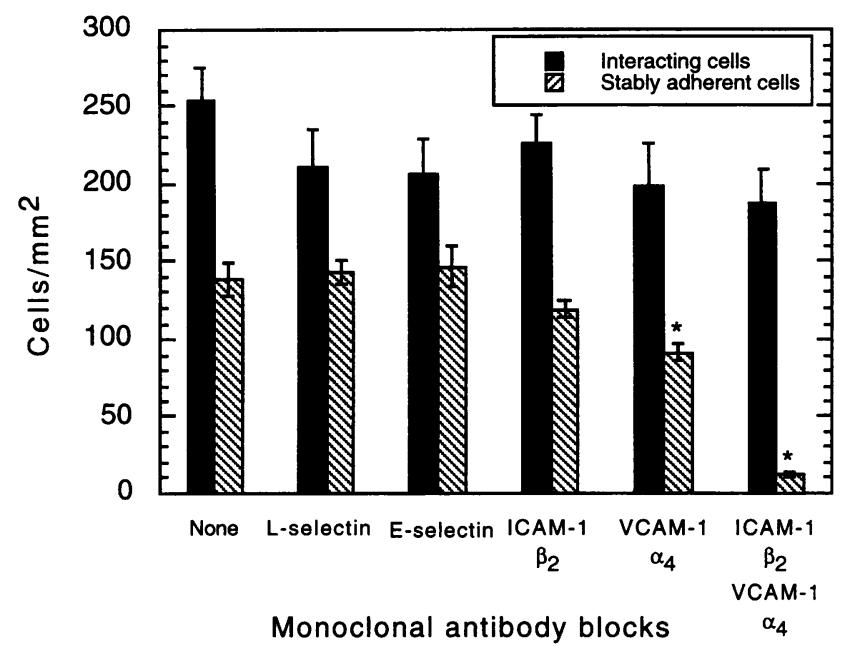

Figure 7. Adhesion of T cells to 24-h IL-1-stimulated HUVECs under flow conditions: effects of receptor-blocking mAbs. None of the mAbs significantly affected the total interacting cells, and blocking both the ICAM- $1 / \beta_{2}$ integrin and VCAM-1/ $\alpha_{4} \beta_{1}$ integrin pathways inhibited stable adhesion of the $\mathrm{T}$ cells by $\sim 90 \%$. In the experiments shown for blockade of L-selectin, the DREG56 mAb was used. However, we also tested a second blocking anti-L-selectin mAb (LAM1-3) and again found no significant difference in adhesion relative to control (data not shown). Control binding was measured with no mAb treatments but we also performed experiments using $\mathrm{mAb}$ PJ-18, which binds an irrelevant HUVEC epitope, and observed similar binding to experiments without mAbs.

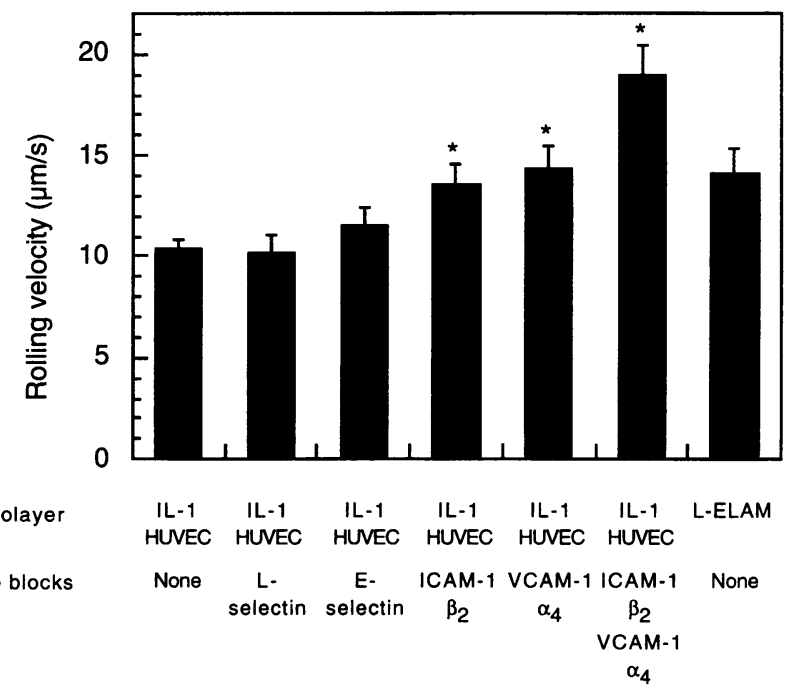

Figure 8. Average rolling velocities of $\mathrm{T}$ cells. Velocities were determined by acquiring 4-s maximization images as shown in Fig. 5. An asterisk indicates a statistically significant $(P<0.05)$ change in rolling velocity relative to no $\mathrm{mAb}$. Values are mean $\pm \mathrm{SEM}$ for $n=40-60 \mathrm{~T}$ cells.

The flow assay revealed important differences in adhesion molecule function by allowing discrimination of primary and secondary adhesive interactions. Similar to what has been reported with neutrophils $(3,5)$, E-selectin transfectant monolayers supported rolling adhesion under flow. L-ICAM-1 monolayers did not support adhesion under flow, even after $\mathrm{T}$ cell stimulation with PMA. Again, these results closely resemble adhesion studies with neutrophils which indicate that $\beta_{2}$ integrins cannot mediate neutrophil adhesion under flow by themselves, but can mediate stable adhesion of activated neutrophils under static conditions or once rolling on EC is initiated by selectin interactions $(1,4,5,8,9)$. Thus, for the E-selectin and $\beta_{2}$ integrin/ICAM-1 pathways, which are shared by $\mathrm{T}$ cells and neutrophils, the adhesion molecules appear to function similarly for both cell types. Because the E-selectin transfectants were able to mediate $\mathrm{T}$ cell adhesion under flow and because this adhesion was exclusively rolling with no long-term stable arrest, E-selectin appears to be able to mediate primary but not secondary adhesion. The L-ICAM-1 cells were unable to support adhesion under flow, but could maintain stable arrest throughout the rinsing period in the static assay, so ICAM-1 appears to be able to mediate secondary but not primary adhesion.

The L-VCAM-1 cells appear to mediate both primary and secondary adhesion of $\mathrm{T}$ cells, since these cells supported binding under flow with $\sim 70 \%$ of the $\mathrm{T}$ cells becoming stably adherent. This binding appeared to be due to $\alpha_{4} \beta_{1} /$ VCAM-1 and not to other possible interactions since control transfectants did not bind lymphocytes and since the observed interaction with VCAM-1 transfectants was completely inhibited by mAbs to VCAM-1 and $\alpha_{4}$ integrins. Moreover, our results are in agreement with preliminary results of another group (Wolber, F., R. Craig, O. Abbassi, J. Ballew, R. Lobb, and L. Stoolman, manuscript in preparation), who observed primary adhesion between an $\alpha_{4} \beta_{1}^{+}$lymphoid tumor cell line and VCAM-1transfected Chinese hamster ovary cells in a similar flow apparatus. It is possible that the ability of $\alpha_{4} \beta_{1}$, but not $\alpha_{\mathrm{L}} \beta_{2}$, to function under flow might be due to differences in the "activa- 
tion" state of these two integrins on resting cells; however, our observation that PMA-treated T cells do not show enhanced primary adhesion to either VCAM-1 or ICAM-1 transfectants (Fig. 3) suggests that the differences are more likely intrinsic to the structure of the adhesion molecules themselves and to the biophysics of their interaction.

Evaluation of $\mathrm{T}$ cell adhesion to HUVEC monolayers suggests that, like neutrophils, $T$ cells use a multistep adhesion cascade utilizing different receptors for primary and secondary adhesion. However, there appear to be differences both in the interaction patterns under flow and in the adhesion pathways contributing to each stage of adhesion. Primary adhesion of T cells to 24-h IL-1-stimulated HUVECs was not limited to a rolling interaction, but also occurred as an immediate arrest of lymphocytes moving with the fluid stream. Primary adhesion in some cases led to secondary adhesion, but in other cases adherent cells detached or continued to roll without stopping. These varied behaviors may reflect functionally distinct subsets of $\mathrm{T}$ cells.

The mechanism(s) responsible for primary adhesion could not be attributed to any member of the selectin family or to VCAM-1. P-selectin was absent from the stimulated HUVECs. E-selectin was expressed at a very low level, and blocking mAbs against E-selectin did not inhibit primary adhesion. Several experiments to test the potential contribution of L-selectin (16, $17,32,33$ ) failed to demonstrate involvement of L-selectin in this system. We also found no contribution of the $\alpha_{4} \beta_{1} /$ VCAM1 pathway to primary adhesion on HUVECs, in contrast to the primary adhesion seen with the L-VCAM-1 transfectants. Primary adhesion to HUVECs stimulated for $24 \mathrm{~h}$ with IL-1 was not significantly affected by anti-VCAM-1 or anti- $\alpha_{4}$ mAbs. HUVECs stimulated with IL-1 + IL-4 displayed even higher levels of VCAM-1 and showed high levels of $\alpha_{4} \beta_{1} /$ VCAM-1dependent secondary adhesion in static assays, yet these EC did not support any primary adhesion of $\mathrm{T}$ cells. The mechanism underlying this difference between the $\mathrm{L}$ cell transfectants and HUVECs remains to be determined.

None of the other adhesion molecules previously implicated in lymphocyte/EC interactions in humans appear likely to account for the primary adhesion observed on 24-h IL-1-treated HUVECs. The lack of inhibition of the anti- $\alpha_{4} \mathrm{mAb}$ HP2/1 would appear to exclude a role for $\alpha_{4} \beta_{7} / \mathrm{MAdCAM}-1$ interactions (11), as this mAb specifically inhibits the $\alpha_{4} \beta_{7}$-dependent binding of human T cells to mouse MAdCAM-1 (Rott, L., and E. Butcher, personal communication). An mAb (Hermes-3) and a polyclonal antiserum against CD44 that have been shown previously to inhibit in vitro lymphocyte-EC interactions (34, 35) also had no effect on the observed interaction (data not shown). The observation that platelet-endothelial cell adhesion molecule-1 is similarly expressed on unstimulated, IL-1-stimulated, and IL-1 + IL-4-stimulated HUVECs suggests that this molecule is not responsible for the primary adhesion. Vascular adhesion protein-1 is not expressed on HUVECs, and lymphocyte-vascular adhesion protein- 2 is constitutively expressed and not inducible on HUVECs, so these recently described adhesion molecules do not appear to play a role in this system $(36,37)$.

Secondary adhesion of T cells to 24-h IL-1 -stimulated HUVEC monolayers appears to be almost completely dependent on the $\alpha_{4} \beta_{1} /$ VCAM-1 and $\beta_{2}$ integrin/ICAM-1 pathways. Additionally, several observations suggest that triggering of a highavidity state of these integrin pathways is an important event in the transition from primary to secondary adhesion. First, stable adhesion was reduced minimally if either integrin pathway was blocked alone, but almost completely if both pathways were blocked, so that under the circumstances of these studies, either pathway appears to provide a sufficient number of receptors for stable adhesion of $T$ cells. Second, evaluation of $T$ cell rolling velocities demonstrated that both sets of integrins contribute to slowing cells which roll, although the interactions are evidently not strong enough to fully stop the cells. Finally, phorbol ester pretreatment, which apparently increases the avidity of binding of both integrin pathways, significantly increased the fraction of interacting cells which adhere stably. Taken together, these observations suggest that for many interacting $T$ cells integrins are initially in a low-avidity state, but for those cells which eventually adhere stably there is a transition to a high-avidity state during which one or both of the integrin pathways take over the majority of the interaction.

The primary adhesion mechanism for $\mathrm{T}$ cell binding to 24h IL-1-stimulated HUVECs appears to be able to function by itself to mediate a rolling interaction, as demonstrated by the experiments with both integrin pathways blocked. The integrin pathways mediating secondary adhesion, however, are apparently unable to function without a primary adhesion mechanism to tether $\mathrm{T}$ cells which are moving with the fluid stream. This is most clearly shown in experiments with combined IL-1 + IL-4 stimulation of HUVEC monolayers, where the primary adhesive mechanism is not evident but the secondary mechanisms develop high levels of adhesion under static conditions. Thus, there is now evidence that $T$ cells can use at least a twostep cascade to adhere to activated ECs under conditions of flow.

\section{Acknowledgments}

This work was supported by National Institutes of Health grants AI31545-01, HL-18672, NS-23327, HL-42550, AI23521, Robert A. Welch Foundation grant C-938, and a grant from the Butcher Fund.

\section{References}

1. Smith, C. W., T. K. Kishimoto, O. Abbassi, B. Hughes, R. Rothlein, L. V. McIntire, E. Butcher, and D. C. Anderson. 1991. Chemotactic factors regulate lectin adhesion molecule 1 (LECAM-1)-dependent neutrophil adhesion to cytokine-stimulated endothelial cells in vitro. J. Clin. Invest. 87:609-618.

2. Jones, D. A., L. V. McIntire, R. P. McEver, and C. W. Smith. 1993. P-selectin supports neutrophil rolling on histamine-stimulated endothelial cells. Biophys. J. 65:1560-1569.

3. Abbassi, O., T. K. Kishimoto, L. V. McIntire, D. C. Anderson, and C. W. Smith. 1993. E-selectin supports neutrophil rolling in vitro under conditions of flow. J. Clin. Invest. 92:2719-2730.

4. Lawrence, M. B., and T. A. Springer. 1991. Leukocytes roll on a selectin at physiologic flow rates: distinction from and prerequisite for adhesion through integrins. Cell. 65:859-873.

5. Lawrence, M. B., and T. A. Springer. 1993. Neutrophils roll on E-selectin. J. Immunol. 151:6338-6346.

6. Tozeren, A., and K. Ley. 1992. How do selectins mediate leukocyte rolling in venules? Biophys. J. 63:700-709.

7. Hammer, D. A., and S. M. Apte. 1992. Simulation of cell rolling and adhesion on surfaces in shear flow: general results and analysis of selectin-mediated neutrophil adhesion. Biophys. J. 63:35-57.

8. Lawrence, M. B., C. W. Smith, S. G. Eskin, and L. V. McIntire. 1990. Effect of venous shear stress on CD18-mediated neutrophil adhesion to cultured endothelium. Blood. 75:227-237.

9. Abbassi, O., C. L. Lane, S. Krater, T. K. Kishimoto, D. C. Anderson, L. V. McIntire, and C. W. Smith. 1991. Canine neutrophil margination mediated by lectin adhesion molecule-1 in vitro. J. Immunol. 147:2107-2115.

10. Shimizu, Y., W. Newman, Y. Tanaka, and S. Shaw. 1992. Lymphocyte interactions with endothelial cells. Immunol. Today. 13:106-112.

11. Berlin, C., E. L. Berg, M. J. Briskin, D. P. Andrew, P. J. Kilshaw, B. Holzmann, I. L. Weissman, A. Hamann, and E. C. Butcher. 1993. Alpha 4 beta 
7 integrin mediates lymphocyte binding to the mucosal vascular addressin MAdCAM-1. Cell. 74:185-195.

12. Weston, S. A., and C. R. Parish. 1990. New fluorescent dyes for lymphocyte migration studies. Analysis by flow cytometry and fluorescence microscopy. J. Immunol. Methods. 133:87-97.

13. Jutila, M. A., T. K. Kishimoto, and M. Finken. 1991. Low-dose chymotrypsin treatment inhibits neutrophil migration into sites of inflammation in vivo: effects on Mac-1 and MEL-14 adhesion protein expression and function. Cell. Immunol. 132:201-214.

14. Picker, L. J., J. R. Treer, B. Ferguson-Darnell, P. A. Collins, P. R. Bergstresser, and L. W. Terstappen. 1993. Control of lymphocyte recirculation in man. II. Differential regulation of the cutaneous lymphocyte-associated antigen, a tissue-selective homing receptor for skin-homing T cells. J. Immunol. 150:11221136.

15. Mulligan, M. S., J. Varani, M. K. Dame, C. L. Lane, C. W. Smith, D. C. Anderson, and P. A. Ward. 1991. Role of endothelial-leukocyte adhesion molecule 1 (ELAM-1) in neutrophil-mediated lung injury in rats. J. Clin. Invest. 88:13961406.

16. Kishimoto, T. K., M. A. Jutila, and E. C. Butcher. 1990. Identification of a human peripheral lymph node homing receptor: a rapidly down-regulated adhesion molecule. Proc. Natl. Acad. Sci. USA. 87:2244-2248.

17. Michie, S. A., P. R. Streeter, P. A. Bolt, E. C. Butcher, and L. J. Picker 1993. The human peripheral lymph node vascular addressin. An inducible endothelial antigen involved in lymphocyte homing. Am. J. Pathol. 143:1688-1698.

18. Berg, E. L., T. Yoshino, L. S. Rott, M. K. Robinson, R. A. Warnock, T. K. Kishimoto, L. J. Picker, and E. C. Butcher. 1991. The cutaneous lymphocyte antigen is a skin lymphocyte homing receptor for the vascular lectin endothelia cell-leukocyte adhesion molecule 1. J. Exp. Med. 174:1461-1466.

19. Picker, L. J., T. K. Kishimoto, C. W. Smith, R. A. Warnock, and E. C. Butcher. 1991. ELAM-1 is an adhesion molecule for skin-homing T cells. Nature (Lond.). 349:796-799.

20. Masinovsky, B., D. Urdal, and W. M. Gallatin. 1990. IL-4 acts synergistically with IL-1 beta to promote lymphocyte adhesion to microvascular endothelium by induction of vascular cell adhesion molecule-1. J. Immunol. 145:28862895.

21. Thornhill, M. H., and D. O. Haskard. 1990. IL-4 regulates endothelial cell activation by IL-1, tumor necrosis factor, or IFN-gamma. J. Immunol. 145:865872 .

22. Thornhill, M. H., S. M. Wellicome, D. L. Mahiouz, J. S. Lanchbury, U. Kyan-Aung, and D. O. Haskard. 1991. Tumor necrosis factor combines with IL4 or IFN-gamma to selectively enhance endothelial cell adhesiveness for T cells. The contribution of vascular cell adhesion molecule-1-dependent and -independent binding mechanisms. J. Immunol. 146:592-598.

23. Briscoe, D. M., R. S. Cotran, and J. S. Pober. 1992. Effects of tumor necrosis factor, lipopolysaccharide, and IL-4 on the expression of vascular cell adhesion molecule- 1 in vivo. Correlation with $\mathrm{CD} 3^{+} \mathrm{T}$ cell infiltration. J. Immunol. 149:2954-2960.

24. Bargatze, R. F., and E. C. Butcher. 1993. Rapid G protein-regulated activation event involved in lymphocyte binding to high endothelial venules. $J$. Exp. Med. 178:367-372.

25. Bjerknes, M., H. Cheng, and C. A. Ottaway. 1986. Dynamics of lymphocyte-endothelial interactions in vivo. Science (Wash. DC). 231:402-405.

26. Lawrence, M. B., L. V. McIntire, and S. G. Eskin. 1987. Effect of flow on polymorphonuclear leukocyte/endothelial cell adhesion. Blood. 70:1284-1290.

27. Watson, S. R. 1993. L-selectin-IgG chimera-in vitro and in vivo. Agents Actions Suppl. 41:103-109.

28. Schmidt, E. E., I. C. MacDonald, and A. C. Groom. 1990. Interactions of leukocytes with vessel walls and with other blood cells, studied by high-resolution intravital videomicroscopy of spleen. Microvasc. Res. 40:99-117.

29. Atherton, A., and G. V. R. Born. 1973. Relationship between the velocity of rolling granulocytes and that of the blood flow in venules. J. Physiol. 233:157165.

30. Ley, K., and P. Gaehtgens. 1991. Endothelial, not hemodynamic, differences are responsible for preferential leukocyte rolling in rat mesenteric venules. Circ. Res. 69:1034-1041.

31. Perry, M. A., and D. N. Granger. 1991. Role of CD11/CD18 in shear rate-dependent leukocyte-endothelial cell interactions in cat mesenteric venules J. Clin. Invest. 87:1798-1804.

32. Spertini, O., F. W. Luscinskas, G. S. Kansas, J. M. Munro, J. D. Griffin, M. A. Gimbrone, and T. F. Tedder. 1991. Leukocyte adhesion molecule-1 (LAM1, L-selectin) interacts with an inducible endothelial cell ligand to support leukocyte adhesion. J. Immunol. 147:2565-2573.

33. von Andrian, U. H., J. D. Chambers, E. L. Berg, S. A. Michie, D. A Brown, D. Karolak, L. Ramezani, E. M. Berger, K. E. Arfors, and E. C. Butcher. 1993. L-selectin mediates neutrophil rolling in inflamed venules through sialy Lewis X-dependent and -independent recognition pathways. Blood. 82:182-191.

34. Oppenheimer-Marks, N., L. S. Davis, and P. E. Lipsky. 1990. Human T lymphocyte adhesion to endothelial cells and transendothelial migration. Alteration of receptor use relates to the activation status of both the $T$ cell and the endothelial cell. J. Immunol. 145:140-148.

35. Jalkanen, S., R. F. Bargatze, J. de los Toyos, and E. C. Butcher. 1987. Lymphocyte recognition of high endothelium: antibodies to distinct epitopes of an $85-95-\mathrm{kD}$ glycoprotein antigen differentially inhibit lymphocyte binding to lymph node, mucosal, or synovial endothelial cells. J. Cell Biol. 105:983-990.

36. Salmi, M., and S. Jalkanen. 1992. A 90-kilodalton endothelial cell molecule mediating lymphocyte binding in humans. Science (Wash. DC). 257:14071409.

37. Airas, L., M. Salmi, and S. Jalkanen. 1993. Lymphocyte-vascular adhesion protein-2 is a novel $70-\mathrm{kDa}$ molecule involved in lymphocyte adhesion to vascular endothelium. J. Immunol. 151:4228-4238. 